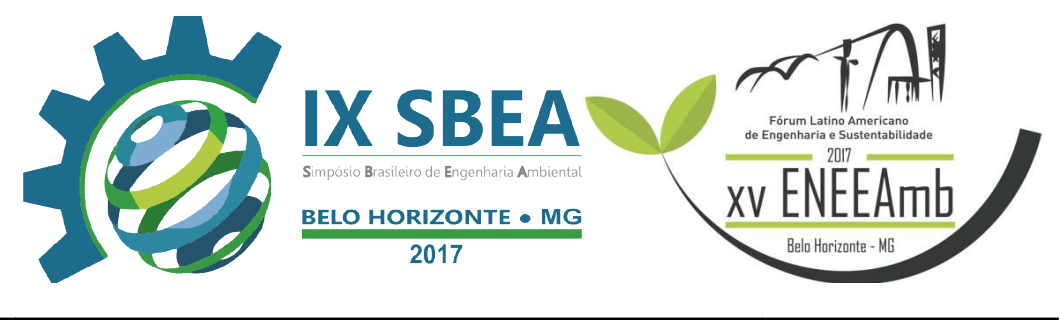

GESTÃO AMBIENTAL E POLÍTICAS PÚBLICAS

\title{
APLICAÇÃO DE INDICADORES DE SUSTENTABILDADE PARA GESTÃO DE RESÍDUOS SÓLIDOS URBANOS
}

Melissa Gurgel de Abreu Machado - melgurgel@gmail.com Instituto Federal de Educação, Ciência e Tecnologia do Ceará

Renan Araujo Almeida - renan.arauju@gmail.com

Instituto Federal de Educação, Ciência e Tecnologia do Ceará 


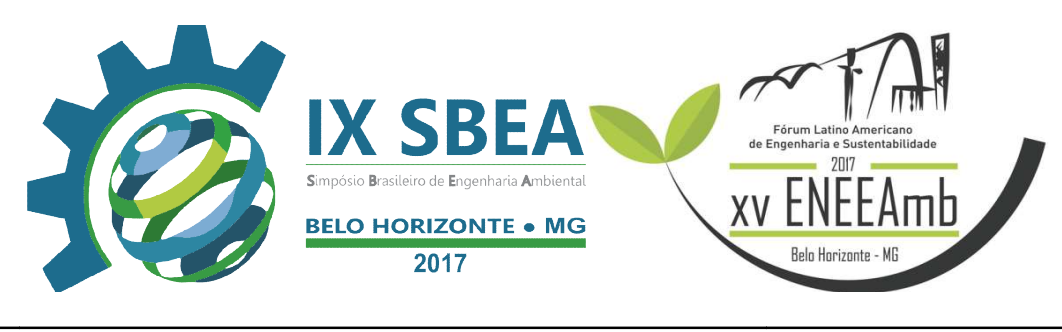

\section{RESUMO}

Devido à maior preocupação com a qualidade de vida da população e do meio ambiente, foi necessária a criação de uma ferramenta capaz de traduzir determinada situação, transmitindo a informação quantitativa que permite o acompanhamento da dinâmica da realidade. Dessa forma, surgiu a aplicação de indicadores de sustentabilidade aplicados a gestão de resíduos sólidos urbanos (RSU). O uso desses indicadores foi indispensável devido a necessidade de um sistema que planeja e executa as políticas públicas de forma eficiente e facilitadora. $\mathrm{O}$ objetivo desse estudo foi mostrar a utilização dos indicadores sustentáveis como ferramentas e a aplicação desses na gestão de RSU. Estudos onde indicadores buscam os princípios da sustentabilidade foram mostrados. O estudo foi baseou-se na utilização de um conjunto de 12 indicadores de sustentabilidade específicos para a gestão de RSU, propostos por MILANEZ (2002) E no artigo técnico feito por Polaz, C e Teixeira, B (2009). Os indicadores propostos são voltados para atender as necessidades dos municípios. Investir em indicadores sustentáveis para o gerenciamento dos RSU é uma forma de buscar a tão necessária sustentabilidade e acompanhar com mais facilidade os problemas enfrentados e as suas soluções.

Palavras-chave: Indicadores de Sustentabilidade, Resíduos Sólidos Urbanos e Gestão de RSU.

\section{INTRODUÇÃO/OBJETIVO}

A partir da década de 90, a questão ambiental, ganhou mais relevância. Sendo observada anteriormente apenas como um fator de preservação do meio ambiente, atualmente a qualidade de vida da população ligada com o meio em que vivemos também está sendo levada em consideração. Portanto, foi gerada uma maior preocupação na forma de gestão, uma preocupação que busca à sustentabilidade. Devido a essa preocupação, foi necessária a criação de sistemas/métodos que permitam traduzir uma situação, analisar e acompanhar a dinâmica da realidade. A partir dessa necessidade, foram criados sistemas de Indicadores de sustentabilidade. Indicadores são ferramentas de informação quantitativa que permitem o acompanhamento da dinâmica 


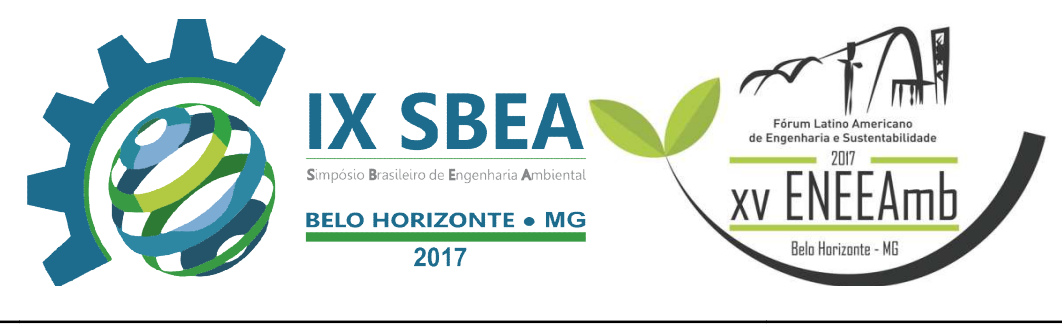

da realidade, sendo utilizados para o monitoramento de uma situação, planejamentos de ações, auxílios e para uma compreensão de dados mais didática. No entanto, para o seu funcionamento, são necessárias uma ou mais unidades de medidas.

Indicadores de sustentabilidade são utilizados para o monitoramento de áreas, avaliação de políticas públicas, auxilio de decisões e facilitam a gestão de forma que mostram a realidade com base em informações pontuais. Dessa forma, auxiliando bastante os administradores públicos, principalmente na área de gestão dos Resíduos Sólidos Urbanos (RSU).

Tendo em vista que quanto maior o consumo, mais resíduos sólidos são gerados e que a maioria dos aglomerados urbanos brasileiros não possuem infraestrutura sanitária para suportar esse crescimento, a gestão dos RSU é um dos maiores problemas socioambientais gerados pela sociedade e enfrentados pelos administradores públicos.

Segundo o pelo Instituto Brasileiro de Geografia e Estatística(IBGE); No Brasil, constitucionalmente, é de competência do poder público local o gerenciamento dos resíduos sólidos produzidos em suas cidades. Segundo a Pesquisa Nacional de Saneamento Básico - PNSB 2008, 61,2\% das prestadoras dos serviços de manejo dos resíduos sólidos eram entidades vinculadas à administração direta do poder público; 34,5\%, empresas privadas sob o regime de concessão pública ou terceirização; e 4,3\%, entidades organizadas sob a forma de autarquias, empresas públicas, sociedades de economia mista e consórcios. Observando-se a destinação final dos resíduos, os vazadouros a céu aberto (lixões) constituíram o destino final dos resíduos sólidos em 50,8\% dos municípios brasileiros, $\begin{array}{lllll}\text { conforme } & \text { revelou } & \text { a } & \text { PNSB } & 2008 .\end{array}$ O objetivo desse estudo foi mostrar a utilização dos indicadores sustentáveis como ferramentas e a aplicação desses na gestão de RSU. Buscando assim mostrar estudos onde os indicadores seguiram os princípios da sustentabilidade.

\section{METODOLOGIA}

A metodologia empregada baseou-se na utilização de um conjunto de 12 indicadores de sustentabilidade específicos para a gestão de RSU, propostos por MILANEZ (2002) - Resíduos sólidos e sustentabilidade: princípios, indicadores e 


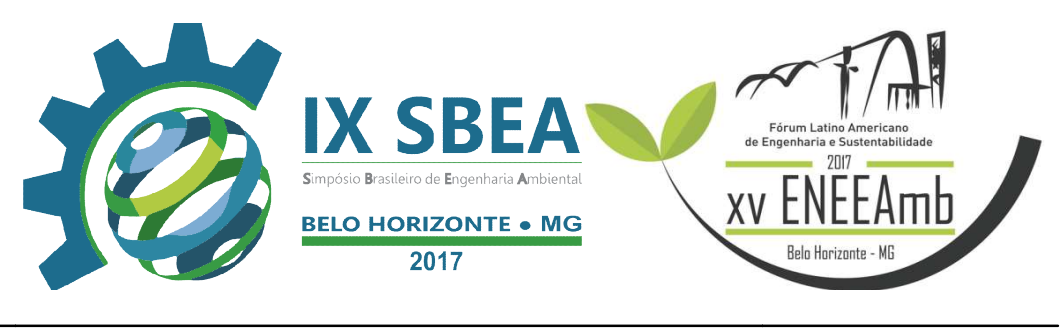

instrumentos de ação - Dissertação (Mestrado em Engenharia Urbana). E no artigo técnico feito por Polaz, C e Teixeira, B (2009) - Indicadores de sustentabilidade para a gestão municipal de resíduos sólidos urbanos: um estudo para São Carlos (SP).

Informações também foram buscadas em revistas cientificas, teses, publicações avulsas. Foram mostrados dados coletados a partir do Instituto Brasileiro de Geografia e Estatística.

\section{RESULTADOS E DISCUSSÃO}

Os indicadores de sustentabilidade para a gestão dos Resíduos Sólidos Urbanos (RSU), são utilizados principalmente servem para auxiliar os gestores públicos na tomada de decisões, em um melhor planejamento de ações e no acompanhamento de tendências.

Para Mourão (2006), pode-se identificar um indicador como uma estatística, um fato, uma medida, uma série quantitativa de dados (indicador quantitativo) ou uma série de evidências ou percepções postuladas sobre a realidade (indicador qualitativo). Já para Soligo aput Takashina (1999), os indicadores são representações quantificáveis das características de produtos e processos, sendo assim utilizados para a melhoria da qualidade e desempenho de um produto, serviço ou processo, ao longo do tempo. Os indicadores surgem como auxiliadores nas tomadas de decisões, fundamentando as argumentações mediante o fornecimento das informações dos processos. Assim, na criação de um indicador, a seletividade, a simplicidade, a clareza, a abrangência, a rastreabilidade, a acessibilidade, a comparabilidade, a estabilidade, a rapidez de disponibilidade e o baixo custo de obtenção são critérios recomendáveis. Para além dos critérios, a criação de um indicador sugere a atribuição de uma meta, a qual consiste na definição de um valor pretendido ao indicador em determinadas condições

De acordo com VAN BELLEN (2005)

Como qualquer outra ferramenta de gestão, os indicadores possuem uma série de limitações técnicas. A maioria dos indicadores relacionados à sustentabilidade não possui um sistema conceitual 


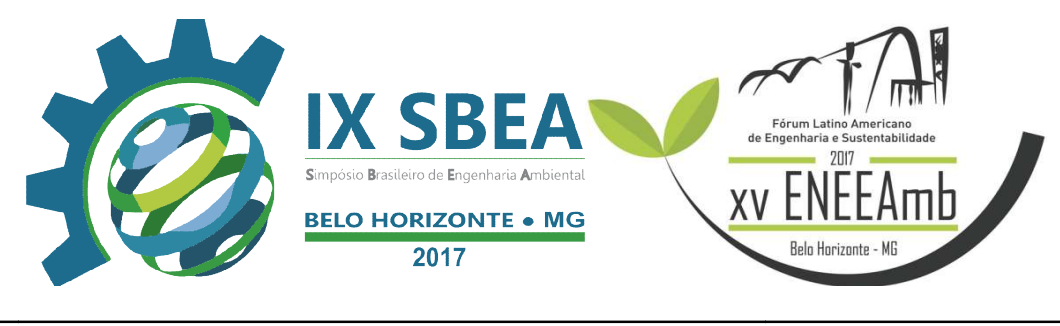

único; medem a aproximação da realidade, e não a realidade precisamente.

Segundo KAYANO e CALDAS (2002)

Além disso, a seleção inadequada de indicadores conduz a um sistema deficiente, muitas vezes ambíguo e, portanto, passível de manipulação política ou de interpretações 'produzidas' ou 'instituídas' da realidade. Logo, antes de utilizá-los, recomenda-se apontar para o aspecto complementar dos indicadores: sua leitura e interpretação devem ser acompanhadas de uma análise minuciosa do fenômeno em questão.

De acordo com POLAZ e TEIXEIRA (2009)

Dentre os indicadores relacionados aos RSU, o indicador mais utilizado no Brasil e no mundo é o da quantidade gerada de resíduos/habitante/unidade de tempo. Outro indicador largamente medido se refere à recuperação de resíduos municipais, percebido como o conjunto de operações (reciclagem, reutilização ou compostagem) que permitem o aproveitamento total ou parcial dos resíduos.

Grimberg (2007) atenta que, para transformar a realidade da gestão de RSU é necessária vontade política por parte dos prefeitos, além da capacitação dos gestores municipais.

Como consequência, tem-se uma avaliação bastante negativa em termos de sustentabilidade, a inexistência de capacitação específica dos funcionários públicos lotados nos setores relacionados à RSU. Em contrapartida, a condição favorável à sustentabilidade seria aquela em que todos os funcionários do setor de RSU estivessem bem preparados tecnicamente. Quando apenas parte do quadro de funcionários recebe algum tipo de capacitação, a tendência é considerada desfavorável à sustentabilidade. 


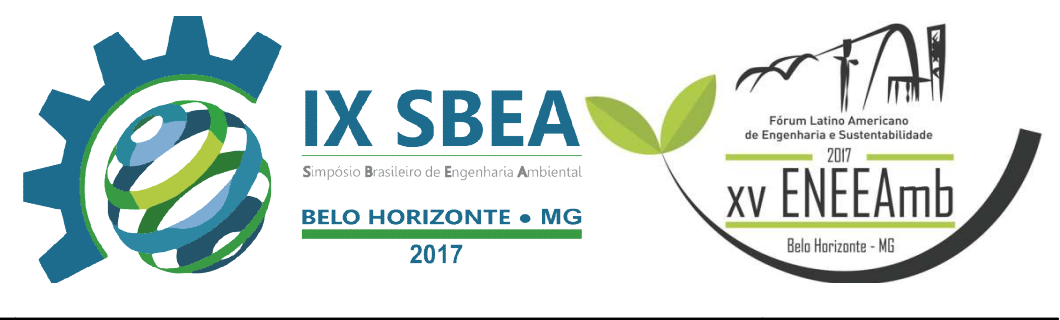

Em 2002, Milanez propôs 12 indicadores de sustentabilidade. Tais indicadores mostrados na tabela 1 são voltados para a gestão pública e municipal de resíduos sólidos urbanos.

Foram definidos três parâmetros de avaliação relativos à tendência à sustentabilidade por Milanez: (I) MD - Muito Desfavorável, (II) D - Desfavorável e (III) F - Favorável.

TABELA 1- Indicadores e avaliação de tendência à sustentabilidade propostos para a gestão de Resíduos Sólidos Urbanos. Fonte: Modificado de MILANEZ (2002).

\begin{tabular}{|c|c|}
\hline INDICADORES DE SUSTENTABILIDADE & $\begin{array}{c}\text { AVALIAÇÃO DE TENDÊNCIA A } \\
\text { SUSTENTABILIDADE }\end{array}$ \\
\hline $\begin{array}{l}\text { (1) Percentual de homens. Dias } \\
\text { efetivamente trabalhados }\end{array}$ & $\begin{array}{l}\text { (MD) Assiduidade inferior a X\% } \\
\text { (D) Assiduidade entre X\% e Y\% } \\
\text { (F) Assiduidade superior a Y\% }\end{array}$ \\
\hline (2) Existência de situações de risco & $\begin{array}{l}\text { (MD) Presença de catadores trabalhando de } \\
\text { forma precária nos locais de disposição } \\
\text { final. } \\
\text { (D) Presença de catadores trabalhando de } \\
\text { forma precária nas ruas. } \\
\text { (F) Inexistência de situações descritas } \\
\text { anteriormente. }\end{array}$ \\
\hline $\begin{array}{l}\text { (3) Percentual das pessoas que atuam na cadeia } \\
\text { de resíduos que têm acesso a apoio ou orientação } \\
\text { definidos em uma política pública municipal }\end{array}$ & $\begin{array}{l}\text { (MD) Inexistência de política pública } \\
\text { municipal efetiva para o apoio às pessoas } \\
\text { que atuam na cadeira de resíduos. } \\
\text { (D) Existência de um programa municipal, } \\
\text { todavia com baixo envolvimento das } \\
\text { pessoas. } \\
\text { (F) Programa municipal de orientação ou } \\
\text { apoio às pessoas que trabalham com } \\
\text { resíduos atingindo um grupo significativo. }\end{array}$ \\
\hline
\end{tabular}

Fonte: MILANEZ (2002) 


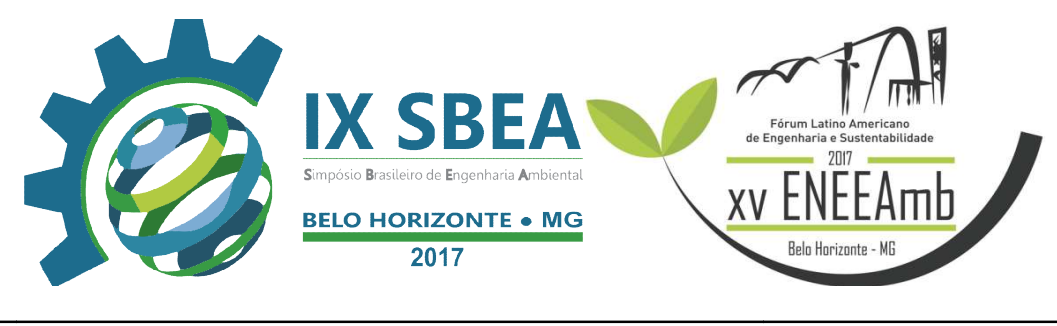

TABELA 1- cont.

\begin{tabular}{|c|c|}
\hline INDICADORES DE SUSTENTABILIDADE & $\begin{array}{l}\text { AVALIAÇÃO DE TENDÊNCIA A } \\
\text { SUSTENTABILIDADE }\end{array}$ \\
\hline $\begin{array}{l}\text { (4) Participação da população através de canais } \\
\text { específicos para gestão dos RSU }\end{array}$ & $\begin{array}{l}\text { (MD) Inexistência dos canais de } \\
\text { participação específicos para RSU. } \\
\text { (D) Existência dos canais de participação } \\
\text { específicos, sem sua utilização pela } \\
\text { população. } \\
\text { (F) Existência de canais específicos e sua } \\
\text { utilização pela população. }\end{array}$ \\
\hline $\begin{array}{l}\text { (5) Existência de parcerias com outras esferas do } \\
\text { poder público ou com a sociedade civil }\end{array}$ & $\begin{array}{l}\text { (MD) Inexistência de parcerias. } \\
\text { (D) Existência de parcerias, mas apenas } \\
\text { dentro do município. } \\
\text { (F) Existência de parcerias tanto dentro } \\
\text { quanto fora do município. }\end{array}$ \\
\hline $\begin{array}{l}\text { (6) Existência de informações sistematizadas } \\
\text { para a população }\end{array}$ & $\begin{array}{c}\text { (MD) As informações não são } \\
\text { sistematizadas. } \\
\text { (D) As informações são sistematizadas, mas } \\
\text { não estão acessíveis à população. } \\
\text { (F) As informações são sistematizadas e } \\
\text { divulgadas de forma proativa para a } \\
\text { população. }\end{array}$ \\
\hline $\begin{array}{l}\text { (7) Percentual da população atendida pela coleta } \\
\text { misturada de resíduos }\end{array}$ & $\begin{array}{l}\text { (MD) Parte da população não é atendida } \\
\text { (D) Toda população é atendida, mas nem } \\
\text { todos regularmente ou na frequência } \\
\text { necessária. } \\
\text { (F) Toda população é atendida na frequência } \\
\text { necessária. }\end{array}$ \\
\hline $\begin{array}{l}\text { (8) Eficiência econômica dos serviços de } \\
\text { limpeza pública (Kg de resíduos } \mathrm{R} \$ 1000,00)\end{array}$ & $\begin{array}{l}\text { (MD) Eficiência econômica não identificada } \\
\text { ou abaixo de R\$ X e R\$ Y. } \\
\text { (D) Eficiência econômica entre R\$ X e R\$ } \\
\text { Y. } \\
\text { (F) Eficiência econômica acima de R\$ Y. }\end{array}$ \\
\hline
\end{tabular}

Fonte: MILANEZ (2002). 


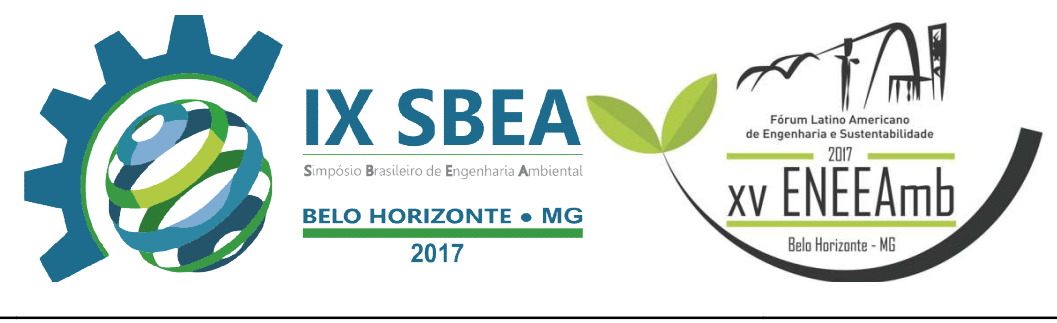

TABELA 1- cont.

\begin{tabular}{|c|c|}
\hline $\begin{array}{l}\text { INDICADORES DE } \\
\text { SUSTENTABILIDADE }\end{array}$ & $\begin{array}{c}\text { AVALIAÇÃO DE TENDÊNCIA A } \\
\text { SUSTENTABILIDADE }\end{array}$ \\
\hline $\begin{array}{l}\text { (9) Percentual autofinanciado do custo de } \\
\text { coleta, tratamento e disposição final }\end{array}$ & $\begin{array}{l}\text { (MD) Não há nenhum sistema de cobrança } \\
\text { para financiamento dos serviços de coleta, } \\
\text { tratamento e destinação final. } \\
\text { (D) Há sistema de financiamento, mas esse } \\
\text { não cobre todos os custos, ou b) há sistema de } \\
\text { financiamento, mas não é proporcional ao uso } \\
\text { dos serviços de coleta, tratamento e destinação } \\
\text { final. } \\
\text { (F) Os serviços de coleta, tratamento e } \\
\text { destinação final são totalmente financiados } \\
\text { pelos usuários proporcionalmente ao uso } \\
\text { desses serviços. }\end{array}$ \\
\hline $\begin{array}{l}\text { (10) Percentual das áreas degradadas pela } \\
\text { gestão dos RSU que já foram recuperadas }\end{array}$ & $\begin{array}{c}\text { (MD) Não foi identificada a existência de } \\
\text { passivo ambiental. } \\
\text { (D) Passivo ambiental identificado, mas sem } \\
\text { recuperação plena. } \\
\text { (F) Passivo ambiental e plenamente } \\
\text { recuperado. }\end{array}$ \\
\hline $\begin{array}{l}\text { (11) Implementação das medidas mitigatórias } \\
\text { previstas nos estudos de impacto ambiental das } \\
\text { atividades relacionadas à gestão dos RSU e } \\
\text { obtenção de licenças ambientais }\end{array}$ & $\begin{array}{l}\text { (MD) Estudos de impacto ambiental não } \\
\text { foram aprovados; não houve licenciamento } \\
\text { ambiental. } \\
\text { (D) Estudos foram aprovados, mas medidas } \\
\text { mitigadoras não foram integralmente } \\
\text { realizadas; houve licenciamento ambiental, } \\
\text { mas não há notificações quanto à não- } \\
\text { conformidades. } \\
\text { (F) Estudos foram aprovados e as medidas } \\
\text { mitigadoras integralmente realizadas; houve } \\
\text { licenciamento ambiental e não há notificações. }\end{array}$ \\
\hline $\begin{array}{l}\text { (12) Percentual, em peso, dos resíduos } \\
\text { coletados pelo poder público e que não são } \\
\text { encaminhados para a disposição final }\end{array}$ & $\begin{array}{l}\text { (MD) Inexistência de programa para } \\
\text { recuperação de RSU. } \\
\text { (D) Recuperação parcial dos materiais } \\
\text { reaproveitáveis presentes nos RSU. } \\
\text { (F) Recuperação significativas dos } \\
\text { materiais reaproveitáveis presentes nos } \\
\text { RSU. }\end{array}$ \\
\hline
\end{tabular}

Fonte: MILANEZ (2002). 


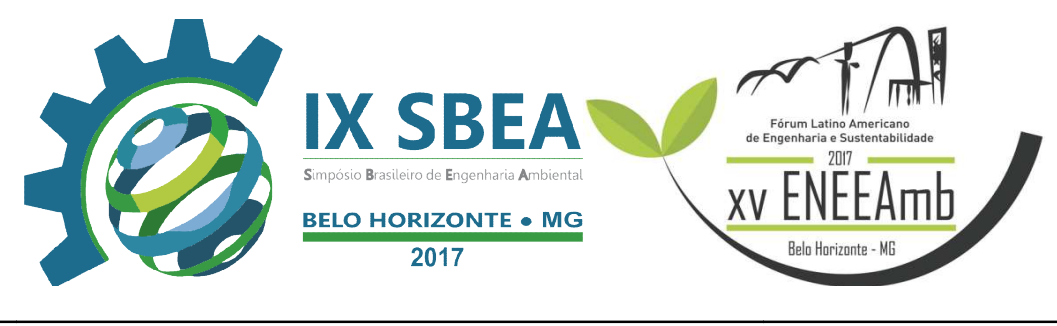

Os princípios adotados que buscam a sustentabilidade em relação aos indicadores propostos por Milanez, são mostrados na tabela 2.

TABELA 2- Princípios para a gestão de Resíduos Sólidos Urbanos

\begin{tabular}{|l|l|}
\hline PRINCÍPIOS PARA RSU & \\
\hline $\begin{array}{l}\text { Garantia decondiçõesadequadas } \\
\text { detrabalho }\end{array}$ & Geração de trabalhoe renda \\
\hline Gestão solidária & Democratização dainformação \\
\hline Universalização dosserviços & Eficiênciaeconômica dagestão dos RSU \\
\hline $\begin{array}{l}\text { Internalização } \\
\text { doscustos e benefícios }\end{array}$ & $\begin{array}{l}\text { Recuperação dadegradaçãoambiental devida } \\
\text { àgestão incorreta dos } \\
\text { RSU }\end{array}$ \\
\hline Previsão deimpactos sócioambientais & Preservação dosrecursos naturais \\
\hline
\end{tabular}

Fonte: MILANEZ (2002).

Com base no estudo de Polaz, C e Teixeira, B(2009), O objetivo principal do trabalho desenvolvido por MILANEZ (2002) foi propor uma estrutura de princípios e indicadores que permitisse avaliar quão sustentável seria um sistema de gestão de RS.

\section{CONCLUSÕES/RECOMENDAÇÕES}

O uso de indicadores de sustentabilidade para a gestão de RSU foi necessária devido a necessidade de um sistema que planeja e executa as políticas públicas de forma eficiente e facilitadora. A gestão desses resíduos é um dos maiores problemas socioambientais enfrentados, pois quanto maior o poder aquisitivo das pessoas, mais resíduos são gerados. É notável a falta de estrutura sanitária na grande maioria dos municípios para o tratamento, destinação, reutilização e reciclagem desses resíduos urbanos desenfreadamente gerados.

Os indicadores se tornaram assim, ferramentas essências para os gestores públicos que buscam um modo de administração mais eficiente e pontual. Esses auxiliam, mas é de suma importância que as prefeituras implementem programas eficientes e planos específicos para os problemas a serem enfrentados. No entanto, é 


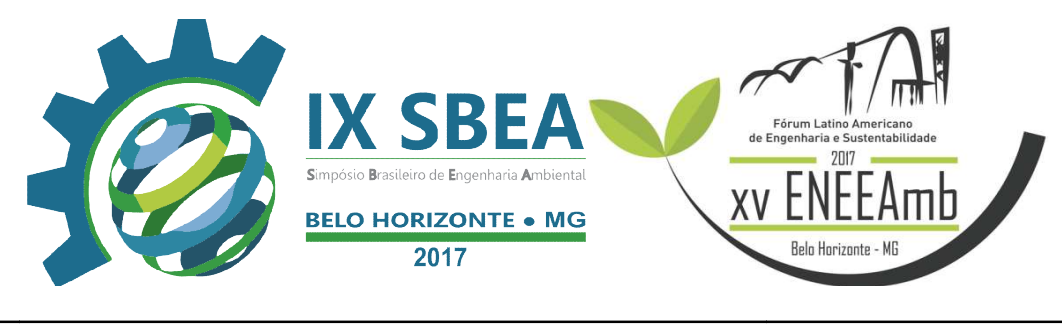

preciso lembrar que existem contradições entre o que os indicadores propõem e o que de fato é realizado.

Os indicadores de sustentabilidade utilizados no estudo de Milanez (2002) são voltados para atender às necessidades dos municípios e para uma melhoria na gestão de RSU.

Investir em indicadores sustentáveis para o gerenciamento dos RSU é uma forma de buscar a tão necessária sustentabilidade e acompanhar com mais facilidade os problemas enfrentados e as suas soluções. Despertando também o interesse da população já que o uso de indicadores facilita o entendimento das informações e a compreensão dos fenômenos que abordam.

É notável que a gestão de RSU não têm sido prioridade para os governos, já que são obras caras e de longo prazo. Isso mostra o quão importante é o uso de indicadores para facilitar o planejamento, avaliar tendências e como ferramenta disseminadora de informação.

\section{REFERÊNCIAS BIBLIOGRÁFICAS}

GRIMBERG, E. Abrindo os sacos de "lixo": um novo modelo de gestão de resíduos está em curso no país. São Paulo, 2007. Disponível em http://www.polis.org.br/uploads/576/576.pdf

Instituto Brasileiro de Geografia e Estatística- Pesquisa Nacional de Saneamento Básico, 2008. Disponível em: http://www.ibge.gov.br/home/estatistica/populacao/condicaodevida/pnsb2008/PNSB_2 008.pdf

KAYANO, J.; CALDAS, E.L. Indicadores para o diálogo. In: CACCIA-BAVA, S.; PAULICA, V.; SPINK, P. (Org.). Novos contornos da gestão local: conceitos em construção. Polis: Programa Gestão Pública e Cidadania. São Paulo: FGV/EAESP, 2002. p. 291-308

MILANEZ, B. Resíduos sólidos e sustentabilidade: princípios, indicadores e instrumentos de ação. 206 f. Dissertação (Mestrado em Engenharia Urbana) Universidade Federal de São Carlos, São Carlos, 2002. Disponível em: http://www.bv.fapesp.br/pt/dissertacoes-teses/4914/residuos-solidos-e-sustentabilidadeprincipios-indicadores-e/:

MOURÃO, P. Contributo para o estudo econômico dos indicadores regionais. Departamento de Economia/Núcleo de Investigação em Políticas Econômicas, 


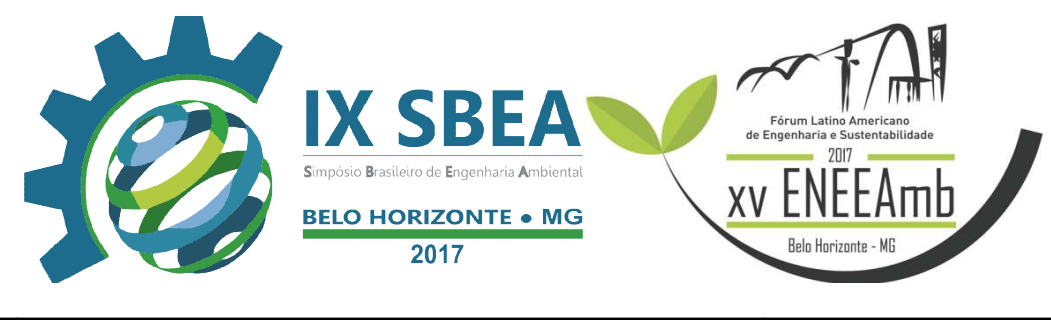

Universidade do Minho, 2006. Disponível em: $<\mathrm{http}$ //www3.eeg.uminho.pt/economia/nipe/docs/Publicações_Outrass_Revistas/com\% 20Arbitragem/2006/Mourão_2006_RPER.pdf;>.

POLAZ, C.; TEIXEIRA, B. Indicadores de sustentabilidade para a gestão municipal de resíduos sólidos urbanos: um estudo para São Carlos (SP) Eng. Sanit. Ambient. vol.14 no.3. Rio de Janeiro July/Sept. 2009. Disponível em: $<$ http://www.scielo.br/scielo.php?script=sci_arttext\&pid=S1413-

41522009000300015;>

SOLIGO, V. Indicadores: Conceito e complexidade do mensurar em estudos de fenômenos sociais. p. 12-25, mai./ago, 2012. Disponível em: $<$ http://www.fcc.org.br/pesquisa/publicacoes/eae/arquivos/1724/1724.pdf; >

TAKASHINA, N. T. Indicadores da qualidade e do desempenho. Rio de Janeiro: Qualitymark, 1999.

VAN BELLEN, H.M. Indicadores de sustentabilidade: uma análise comparativa. Rio de Janeiro: FGV, 2005. Disponível em: $<$ http://books.google.com.br/books?hl=pt$\mathrm{BR} \& 1 \mathrm{r}=\& \mathrm{id}=\mathrm{EKPPu} 5 \mathrm{y} 5 \mathrm{WyIC} \& \mathrm{oi}=\mathrm{fnd} \& \mathrm{pg}=\mathrm{PA} 72 \& \mathrm{ots}=\mathrm{vW} 4 \mathrm{APx} 1 \mathrm{fLc} \& \operatorname{sig}=\mathrm{C} 175 \mathrm{Bkd}$ WBCPrZiNQY-mwZPmIHkc\&redir_esc $=\mathrm{y} \# \mathrm{v}=$ onepage \&q\&f=false ;> 